\title{
The Dynamic Properties of a Perturbation System
}

\author{
Lizhen Zhang \\ Department of Mathematics \\ Tianjin Polytechnic University \\ Tianjin 300160, China \\ E-mail:lzhzhang@hotmail.com \\ Li Jia \\ Department of Mathematics \\ Tianjin Polytechnic University \\ Tianjin 300160, China \\ E-mail:jiali0@yahoo.cn
}

\begin{abstract}
In this paper,we study the dynamics of a perturbation system. Firstly, we consider the unperturbation system and give the types of the fixed points of the system by nullcline. Then we analysis the Dynamic behavior of the orbits around the fixed points. Further we study the dynamics of the perturbation system using the Melnikov methods, which possess some universality.
\end{abstract}

Keywords: Nullcline, Phase portrait, Melnikov Function, Manifold

\section{Introduction}

In recent years, Dynamical systems have had applications in science and engineering, more and more people recognize the nonlinearity, complexity and systematicness of the world. From the motion of the heavenly body to the movement of the microscopic particle all show the nonlinearity, complexity and systematicness(Huang,2000). So the study for the action of the nonlinear systems is very important. In this paper, we study a nonlinear perturbation system(Liu,1994;S,1990,). The system is

$$
\left\{\begin{array}{l}
\dot{x}=y \\
\dot{y}=x-x^{3}-\varepsilon(y+f(x))
\end{array}\right.
$$

where $(x, y) \in R^{2}, f(x)$ is a continuous function, $0<\varepsilon \ll 1$.

When $\varepsilon=0$, the unperturbation system of (1) is

$$
\left\{\begin{array}{l}
\dot{x}=y \\
\dot{y}=x-x^{3}
\end{array}\right.
$$

First we consider the unperturbation system and give the types of the fixed points of the system by nullcline of the orbits of the system (2) and analyze the Dynamic behavior of the orbits around the fixed points, that is phase portrait . Further we study the dynamics of the perturbation system using the Melnikov methods, this methods possess some universality.

\section{The phase portrait of the unperturbation system}

In this paper, we will regard the ordinary differential equation possessing perturbation (1). Firstly, we think the unperturbation system of the system (1), that is (2).

From

$$
\left\{\begin{array}{l}
\dot{x}=0 \\
\dot{y}=0
\end{array}\right.
$$

We have

$$
\left\{\begin{array}{l}
y=0 \\
x-x^{3}=0
\end{array}\right.
$$


Thus we know the system (2) has three fixed point(J,1999): $O(0,0), A(-1,0), B(1,0)$. We will study the vector field of the three fixed points by the nullcline of the orbits of the system (2). From this we can judge the types of these fixed points.

From (3), $y=0, x=0, x= \pm 1$ are four nullclines of the system (2). The four nullclines are divided into ten partitions each other. The ten partitions are:

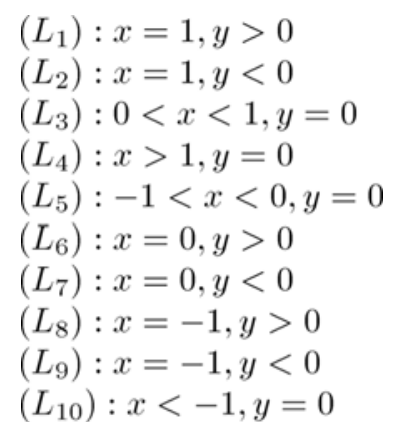

And the four nullclines divide the plane $x O y\left(R^{2}\right)$ into eight districts (the ten partitions are not included), that is

$\left(A_{1}\right): x>1$ and $y>0$

$\left(A_{2}\right): x>1$ and $y<0$

$\left(A_{3}\right): 0<x<1$ and $y>0$

$\left(A_{4}\right): 0<x<1$ and $y<0$

$\left(A_{5}\right):-1<x<0$ and $y>0$

$\left(A_{6}\right):-1<x<0$ and $y<0$

$\left(A_{7}\right): x<-1$ and $y>0$

$\left(A_{8}\right): x<-1$ and $y<0$

In order to confirm the direction of the orbits, we consider the nullclines of the ten partitions and the 8 districts . On the $\dot{x}=0$, the nullcline is vertical. And on the $\dot{y}=0$, the nullcline is horizontal (see figure 1). Because the situations is similarity, we only consider direction of the orbits through the half-line $L_{1}: x=1, y>0$. In this situation, $x=1, y>0$, we have $\dot{x}>0, \dot{y}=x-x^{3}=0$, so we can judge the direction of the orbits is horizontal to the right (see figure 1). On the left of the half-line $L_{1}: x=1, y>0$, we have $0<x<1$, then $\dot{y}<0, \dot{x}>0$, $\frac{\dot{y}}{\dot{x}}>0$. This is the direction of the orbits in district $A_{3}$ (see figure 1). The discussions of the districts $A_{1}, A_{2}, A_{4}-A_{8}$ are similar to the district $A_{3}$. So we have the following figure 1.

The ten partitions of divided by the four nullclines and the vector fields on the nullclines are:

$\left(L_{1}\right): x=1, y>0$, horizontal, $\dot{x}>0$, to the right

$\left(L_{2}\right): x=1, y<0$, horizontal, $\dot{x}>0$, to the left

$\left(L_{3}\right): 0<x<1, y=0$, vertical, $\dot{y}>0$, up

$\left(L_{4}\right): x>1, y=0$, vertical, $\dot{y}<0$, down

$\left(L_{5}\right):-1<x<0, y=0$, vertical, $\dot{y}<0$, down

$\left(L_{6}\right): x=0, y>0$, horizontal, $\dot{x}>0$, to the right

$\left(L_{7}\right): x=0, y<0$, horizontal, $\dot{x}<0$, to the left

$\left(L_{8}\right): x=-1, y>0$, horizontal, $\dot{x}>0$, to the right

$\left(L_{9}\right): x=-1, y<0$, horizontal, $\dot{x}<0$, to the right

$\left(L_{10}\right): x<-1, y=0$, vertical, $\dot{y}>0$, up

The eight areas of divided by the four nullclines and the vector fields in these areas are:

$\left(A_{1}\right): x>1$ and $y>0, \dot{y}<0$ and $\dot{x}>0$

$\left(A_{2}\right): x>1$ and $y<0, \dot{y}<0$ and $\dot{x}<0$

$\left(A_{3}\right): 0<x<1$ and $y>0, \dot{y}>0$ and $\dot{x}>0$

$\left(A_{4}\right): 0<x<1$ and $y<0, \dot{y}>0$ and $\dot{x}<0$

$\left(A_{5}\right):-1<x<0$ and $y>0, \dot{y}<0$ and $\dot{x}>0$

$\left(A_{6}\right):-1<x<0$ and $y<0, \dot{y}<0$ and $\dot{x}<0$

$\left(A_{7}\right): x<-1$ and $y>0, \dot{y}>0$ and $\dot{x}>0$

$\left(A_{8}\right): x<-1$ and $y<0, \dot{y}>0$ and $\dot{x}<0$

From these, we can judge the fixed point $O(0,0)$ is a saddle point , $A(1,0), B(-1,0)$ are center point or focus . Of course we judge $A(1,0), B(-1,0)$ are center points of the system (2). So we can describe the phase portrait of the system (2) (see figure 2): 


\section{The Dynamics of the perturbation system}

When $0<\varepsilon \ll 1$, the dynamics of the system (1) is complexity, the period orbits and the rotation period orbits around the saddle point $O$ and center points $A, B$ of the system (2) are possibly rupture, forming stable and unstable manifolds. Following we judge the dynamics of the orbits by Malenkov methods [2].

Suppose

$$
\left\{\begin{array}{l}
P=y \\
Q=x-x^{3}-\varepsilon(y+f(x))
\end{array}\right.
$$

We have

$$
\frac{\partial P}{\partial x}+\frac{\partial Q}{\partial y}=-\varepsilon<0
$$

By Benison criterion [3], we conclude that there doesn't exist closed orbits. So from there exist the small perturbation $-\varepsilon(y+f(x))$, the period orbits, homoclinic orbits and the rotation period orbits of (2) are all rupture . We must judge the change of the stable and unstable manifolds by Melnikov methods. Because the perturbation $(0,-(y+f(x)))$ is autonomous, the Melnikov function of the orbits $q_{0}(t)$ of (1) is

$$
\begin{aligned}
M\left(t_{0}\right) & =\int_{-\infty}^{+\infty} q_{0}(t) \wedge g\left(q_{0}(t)\right) d t \\
& =\int_{D} \int\left(\frac{\partial g_{1}}{\partial x}+\frac{\partial g_{2}}{\partial x}\right) d x d y \\
& =-\int_{D} \int d x d y \\
& =-S_{D}<0
\end{aligned}
$$

where $g=\left(g_{1}, g_{2}\right), g_{1}=0, g_{2}=-(y+f(x)), D$ is the Area from one side $x>0$ of the orbits of (1). From $M\left(t_{0}\right)<0$, we can conclude the unstable manifolds which are included the stable manifolds. The orbits is showed in figure 3:

\section{Conclusion}

Judging the direction of the orbits by the nullcline is a good method, which is simple and clear .The Melnikov methods of orbits is maneuverability. This is rigorously analysis methods and this method is used others fields.

\section{References}

Huang Runsheng, Chaos and Application, Wuhan University Press, Wuhan, (2000).

J.Guckenheimer and P.holmes, Nonlinear Oscallators, Dynamical Systems, and Biffurcations of Vector Fields.Springer, (1999).

Liu Zengrong, Perturbation Criteria For Chaos, Shanghai Scientific and Technological Education Publishing House, Shanghai, (1994).

S.Wiggins, Introduction to applied nonlinear Dynamical Systems and Chaos, Spring-Verlag, (1990).

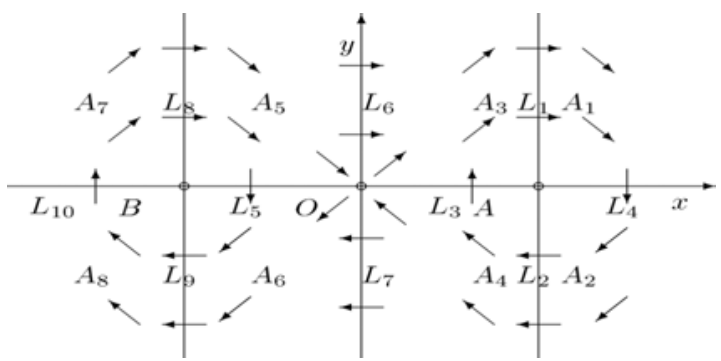

Figure 1. The nullclines of the system (2) 


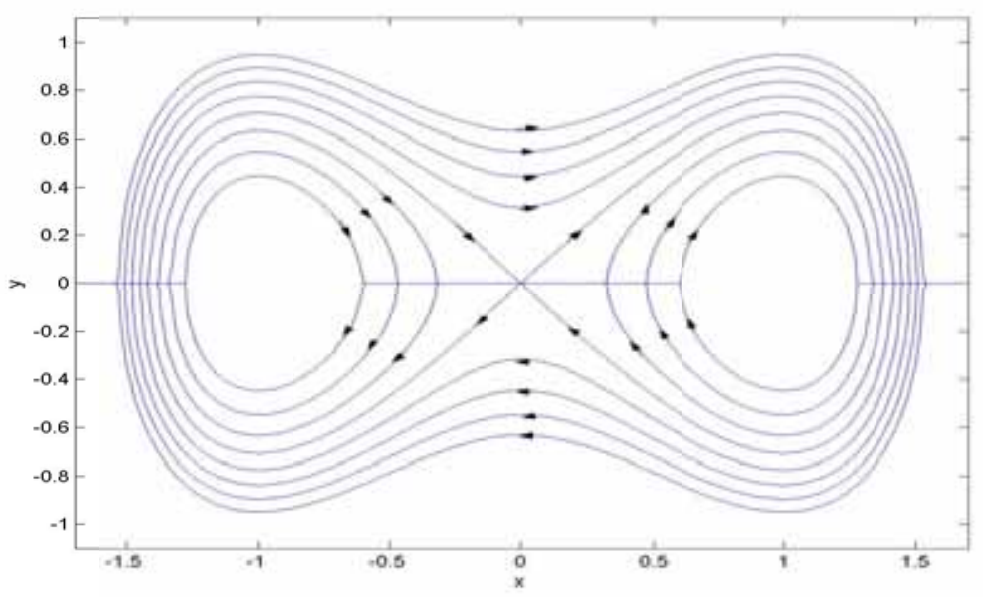

Figure 2. the phase portrait of the system (2)

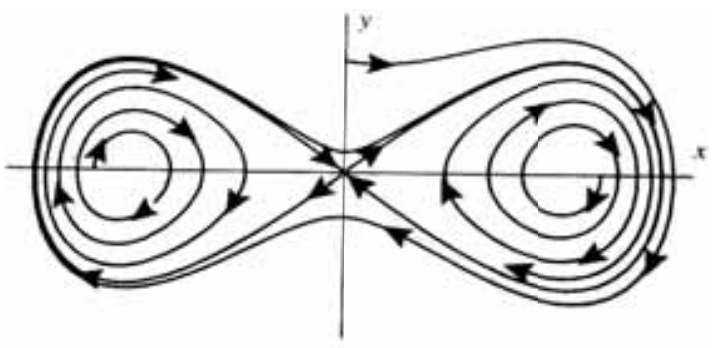

Figure 3. The orbits portrait of the perturbation system (1) 